\title{
Jun in Drosophila development: redundant and nonredundant functions and regulation by two MAPK signal transduction pathways
}

\author{
Lutz Kockel, Julia Zeitlinger, Lena M. Staszewski, Marek Mlodzik, ${ }^{1,2}$ and Dirk Bohmannn ${ }^{1,2}$ \\ European Molecular Biology Laboratory, (EMBL), D-69117 Heidelberg, Germany
}

\begin{abstract}
Drosophila Jun is shown to be involved in different signal transduction pathways and developmental decisions. Dorsal closure, a morphogenetic process occurring during Drosophila embryogenesis, is regulated by Hemipterous (Hep) and Basket (Bsk), homologs of JNKK and JNK, respectively. Embryos lacking Jun activity exhibit a dorsal closure phenotype, very similar to that of bsk and hep mutants, indicating that Jun is a target of Hep/Bsk signaling. In eye and wing development Jun participates in a separate signaling pathway that is comprised of Ras, Raf, and the ERK-type kinase Rolled. In contrast to the strict requirement for Jun in dorsal closure, its role in the eye is redundant but can be uncovered by mutations in other signaling components. The redundant function of Jun in eye development may contribute to the precision of photoreceptor differentiation and ommatidial assembly.
\end{abstract}

[Key Words: Drosophila development; Jun; JNK; ERK; signal transduction; transcription factor]

Received March 17, 1997; revised version accepted May 5, 1997.

The paramount importance of cellular signal transduction pathways is particularly evident in multicellular organisms. These pathways not only mediate communication between cells but also processing of the exchanged information. In this manner they control complex functions during morphogenesis as well as in the adult organism. As the charting of signal transduction pathways proceeds toward an understanding in molecular detail, certain unexpected features emerge: At least some signaling components are used in more than one pathway. The small GTPase Ras, for example, is an element of growth factor response pathways but also of pathways that control differentiation of postmitotic cells (Zipursky and Rubin 1994; Marshall 1995). Likewise, many transcription factors such as Elk, cAMP response element binding (CREB) protein and NF- $\mathrm{kB}$ are receptive to stimuli from a number of different pathways (Armstrong and Montminy 1993; Darnell et al. 1994; Siebenlist et al. 1994; Treier and Bohmann 1996).

Another recently discovered feature of cellular signaling is the existence of pathways that have quite different biological functions but consist of homologous components. The best-known examples are the different mitogen-activated protein kinase (MAPK) signaling path-

\footnotetext{
${ }^{1}$ These authors contributed equally to this work and are co-senior authors.

${ }^{2}$ Corresponding authors.

E-MAIL mlodzik@embl.heidelberg.de; bohmann@embl.heidelberg.de; FAX 00496221387306.
}

ways, first identified in yeast. The stimuli that lead to specific yeast responses, such as mating behavior after pheromone exposure or the reaction to osmotic stress, are relayed by distinct, yet related signal transduction pathways (Herskowitz 1995). The hallmark of these different pathways are cascades of at least three kinases which are now classified as MAPKKK, MAPKK, and MAPK (Marshall 1994).

Multiple functions of individual signaling components and interconnections of different signaling pathways indicate that the regulatory network that controls the behavior of cells in the context of multicellular organisms is very complex. Genetic studies in comparatively simple organisms have proved valuable in unraveling this complexity. Studies in Drosophila melanogaster and in Caenorhabditis elegans have contributed significantly to the deciphering of the Ras and other signal transduction pathways (Greenwald and Rubin 1992; Zipursky and Rubin 1994), and it may be assumed that genetics in combination with classic biochemical approaches will lead to a more complete understanding of regulatory networks.

Nuclear elements of cellular signal transduction include inducible transcription factors such as Jun. Jun activity responds to multiple cues such as growth factors, phorbol esters, activated oncogenes such as ras, and cellular stress (Angel and Karin 1991). Previously, we have studied the role of Jun as a nuclear target of the Ras/Raf/ MAPK signal transduction pathway in Drosophila eye 
development (Zipursky and Rubin 1994). Transgene expression studies employing a panel of dominant-negative and constitutively activated mutants showed that Jun participates in the control of photoreceptor determination and differentiation during eye development (Bohmann et al. 1994, Treier et al. 1995; Peverali et al. 1996). Experiments in which the loss of R7 photoreceptors in mutants lacking the Sevenless (Sev) receptor, a tyrosine kinase, could be rescued by expression of activated jun alleles illustrated the potential of Jun to initiate neuronal differentiation in the developing eye (Treier et al. 1995). Biochemical data indicate that the neurogenic function of Jun depends on its phosphorylation by the extracellular signal-regulated kinase (ERK)-type MAPK Rolled (R1), a genetically defined downstream element of Ras signaling (Peverali et al. 1996).

Based primarily on mammalian tissue culture experiments, a related MAPK signal transduction pathway has been identified recently (Davis 1994). The MAPKs activated by this pathway are called JNKs (for Jun amino (N)-terminal kinase) or SAPKs (for stress-activated protein kinase) and are distantly related to ERK-type MAPK (Dérijard et al. 1994; Kyriakis et al. 1994). In vertebrate cells JNK signaling mediates cellular stress responses elicited, for example, by UV radiation, heat shock, and DNA damage (Minden et al. 1994). The relationship of ERK and JNK signaling and their relevance in a whole organism are understood only poorly.

The identification of the product of the hemipterous (hep) gene as a $D$. melanogaster JNKK homolog (Glise et al. 1995) and of basket (bsk) as a JNK (Riesgo-Escovar et al. 1996; Sluss et al. 1996) has made a genetic approach to investigate the JNK pathway possible. Phenotypic analysis in the embryo, as well as biochemical data, indicate that Bsk and Hep are part of a Drosophila JNK signal transduction cascade (Knust 1996). Mutations in bsk and hep cause almost identical phenotypes. In both cases, mutant embryos fail to complete dorsal closure, a morphogenetic process occurring in late embryonic stages (Campos-Ortega and Hartenstein 1985). During dorsal closure the cells of the lateral epidermis undergo a concerted shape change and elongate along the dorsoventral axis. This process, which occurs in the absence of cell division, results in a dorsalward movement of the lateral epidermis enclosing gut and mesodermal derivatives, which until then are only covered by the amnioserosa. The cell shape changes of dorsal closure initiate in the cells of the leading edge of the lateral epidermis and may be controlled by a signal emanating from it (Knust 1996). Biochemical data indicate that Hep can phosphorylate Bsk, supporting the hypothesis that the two proteins are components of the same signal transduction pathway. It was also suggested by in vitro phosphorylation experiments that Bsk can phosphorylate Drosophila Jun (Riesgo-Escovar et al. 1996; Sluss et al. 1996). This might indicate that as in mammals, Jun is a target of the JNK signal transduction pathway in $D$. melanogaster. If true, this would provide a model in which the function of Jun as a target of both ERK and JNK signaling could be studied in vivo in the same organism.
Here we show that Drosophila Jun acts in multiple developmental control processes as a target of both ERK and JNK signaling. Our data indicate that Jun plays an essential role in dorsal closure, whereas in eye development, loss of Jun in an otherwise wild-type background can be tolerated with only minor phenotypic defects. Nevertheless, Jun becomes a limiting component if ERK signaling is reduced, demonstrating a redundant function of Jun in Drosophila similar to that proposed in mammals (Hilberg et al. 1993; Johnson et al. 1993).

\section{Results}

To investigate whether Jun might be involved in the Hep/Bsk signaling pathway in the control of dorsal closure, we followed three experimental strategies: (1) immunostaining of embryos for Jun protein in the relevant cells; (2) expression of dominant-negative forms of Jun in the relevant embryonic tissues; and (3) analysis of mutant embryos that lack a functional jun gene.

\section{Jun expression in the early embryo}

First, we asked whether the temporal and spatial expression pattern of Jun was compatible with a role in dorsal closure. Whole-mount immunostaining of wild-type Drosophila embryos with affinity-purified Jun antibodies (Bohmann et al. 1994; Peverali et al. 1996) reveals widespread nuclear Jun expression at varying levels throughout embryogenesis. One of the tissues showing elevated Jun expression is the amnioserosa (Fig. 1A). During and following germ-band retraction, cells of the dorsolateral epidermis also show increased Jun staining (Fig. 1B-D). Strikingly, highest Jun protein levels are detected in the cells at the leading dorsal edge of the epidermis. These cells initiate the process of dorsal closure and are also the first that undergo the associated cell shape changes (an elongation along the dorsoventral axis). The high expression of Jun in the cells of the leading edge of dorsal closure is maintained throughout this process until its completion in late embryogenesis (Fig. $1 \mathrm{E}, \mathrm{F})$. Cells along the cephalic furrow also express Jun at high levels (arrowheads in Fig. 1C,E).

The elevated staining in the leading edge of the lateral epidermal walls where dorsal closure initiates is consistent with a role for Jun in the process and lends credence to the hypothesis that Jun might be a target for the Hep/ Bsk signaling pathway.

\section{Dominant-negative forms of Jun interfere with dorsal closure}

To obtain functional evidence for the involvement of Jun in dorsal closure, we employed a previously characterized dominant-negative mutant in which the transcriptional activation domains are deleted (Jun ${ }^{\text {hZIP }}$ ) (Bohmann et al. 1994). This truncated derivative was expressed in the epidermis at relevant embryonic stages using the upstream activating sequence (UAS)-Gal4 expression system (Brand and Perrimon 1993). Embryonic expression of 


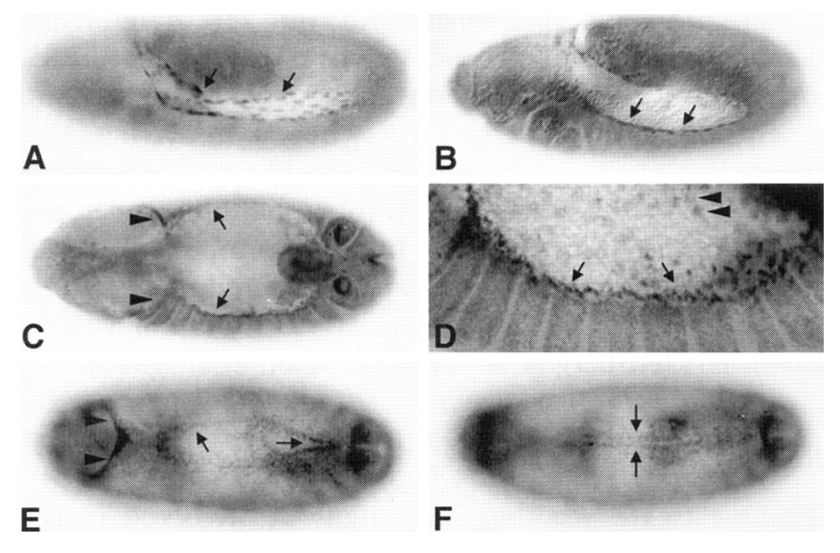

Figure 1. Jun expression in embryos during dorsal closure. Wild-type embryos were stained with an affinity-purified polyclonal antiserum raised against bacterially expressed Drosophila Jun. All embryos are oriented anterior left; $(A, B, D)$ Lateral views (dorsal up); $\{C, E, F)$ dorsal views. Stages are according to Campos-Ortega and Hartenstein (1985). (A) Stage 10: Note elevated nuclar expression in cells of the amnioserosa (arrows). (B) Stage 11: Transiently high levels of Jun are detected in the cells of the amnioserosa that are directly adjacent to the dorsolateral epidermis (arrows). Lower levels of Jun staining remain present in all cells of the amnioserosa throughout their life time $(D$; data not shown). $(C, D)$ Early stage 13 : High Jun expression levels are detected in the cells of the leading edge of the lateral epidermis (arrows) and cells posteriorly adjacent to the head segments (arrowheads in C), which will undergo head involution. Note that lower levels of Jun are detectable in all cells of the lateral epidermis, and cells of the amnioserosa show also lower levels of Jun at this and later stages (arrowheads in $D$; data not shown). (E) Stage 15: Note that expression is maintained in cells of the leading edge of dorsal closure (arrows) and cells posteriorly adjacent to the head segments (arrowheads). (F) Stage 16: High Jun expression is maintained at the leading edge (arrows); dorsal closure is complete. Jun is also expressed in many other tissues and cell types throughout embryogenesis (not shown).

Jun ${ }^{\text {bZIP }}$ is lethal and causes the appearance of large holes in the dorsal epidermis, which are indicative of a failure in dorsal closure (Fig. 2). Cuticle preparations of these embryos display very similar phenotypes compared to embryos that are homozygous for loss-of-function alleles of bsk or hep. Taken together with the previously reported biochemical data (Riesgo-Escovar et al. 1996; Sluss et al. 1996), the resemblance of the dorsal closure phenotypes caused by hep, bsk, and dominant-negative Jun suggests that the function of Jun in dorsal closure is that of a nuclear recipient of the Drosophila JNK signal transduction pathway. This hypothesis is further supported by the observation that Jun expression is not affected in bsk mutants. In zygotic $b s k^{2}$ homozygotes the Jun protein patterns and levels appear largely normal (not shown).

\section{A jun null mutation disrupts dorsal closure}

To obtain further proof for the role of Jun in dorsal closure, we wanted to examine the effect of endogenous mutations in the Drosophila jun locus during embryogenesis. As a first step toward obtaining loss-of-function jun alleles, we generated a small deletion by imprecise excision of P-element 1(2)07103, which is inserted $2.8 \mathrm{~kb}$ downstream of the jun transcription unit (Fig. 3). This deletion causes a cytologically nonvisible deficiency termed E73 that, like 1(2)07103 (Kockel et al. 1997), is a lethal mutation. E73 completely removes the jun locus, as well as the first exon of the neighboring 14-3-3 $\zeta$ gene (Kockel et al. 1997). Based on the assumption that lack of jun would cause a lethal phenotype, we screened for ethylmethane sulfonate (EMS)-induced point mutations that failed to complement the lethality of E73 (see Materials and Methods). Among the isolated mutations, one jun allele, henceforth referred to as jun ${ }^{1}$ was identified. As determined by PCR and sequence analysis, this allele carries a nonsense mutation at codon 177 of the jun open reading frame (Fig. 3). Because of the stop codon at this position, iun ${ }^{1}$ encodes a truncated protein devoid of the
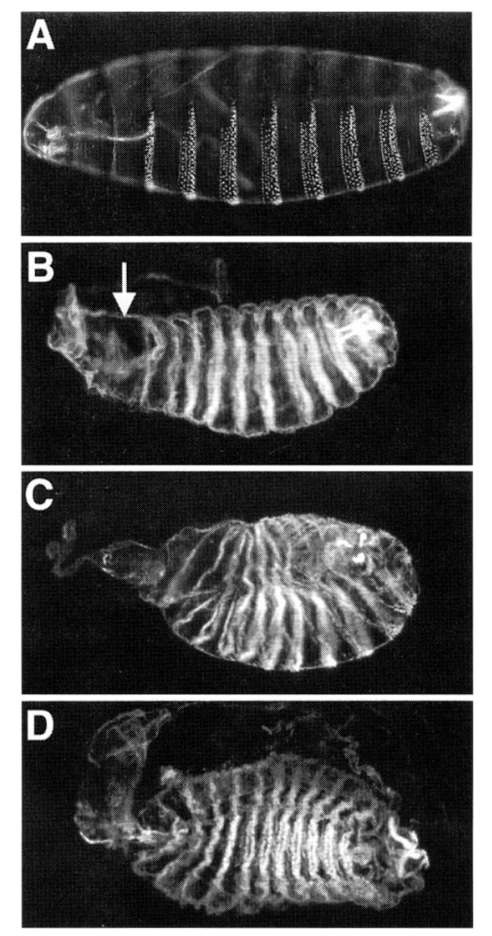

Figure 2. Expression of dominant negative Jun in the developing embryo can cause defects in head and dorsal cuticle development. The dominant-negative Jun ${ }^{\text {bZIP }}$ protein was expressed using the GAL4/UAS system with different embryonic epidermal GAL4 driver lines. Cuticle preparations of the following genotypes are shown: $(A)$ Wild type; $(B) C 765 / U A S$-jun ${ }^{b Z I P} ;(C)$ $69 \mathrm{~B} / U A S-j u n^{b Z I P} ;(D)$ hsGAL4/UAS-jun ${ }^{b Z I P}$. Anterior is left and dorsal up except in $D$, which is a dorsal view. Note that all drivers in combination with $U A S-j u n^{b Z I P}$ cause defects in head and dorsal cuticle and that they display graded severity: The C765 line induces predominantly an anterior open-like phenotype (marked with arrow), whereas 69B and hsGAL4 (following heat shock) lead to phenotypes that progressively affect the entire dorsal cuticle. No defects in posterior structures, the Filzkörper, are observed. 


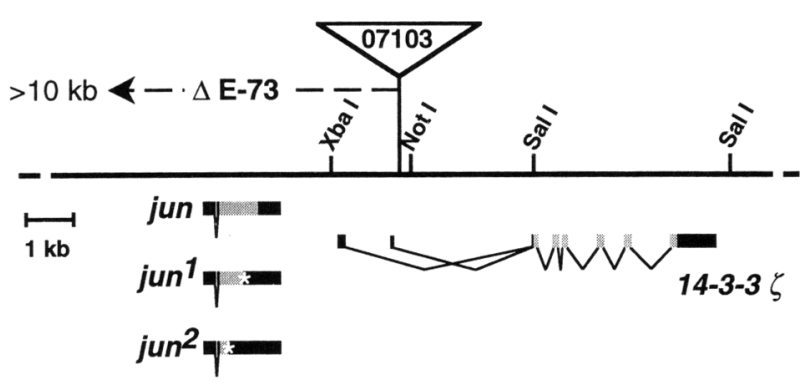

1 MKTPVSAAAN LSIONAGSSG ATAIOIIPKT EPVGEEGPMS LDFQSPNLNT * jun ${ }^{2}$

SNNLMQTPQP GKVFPTKAGP VTVEQLDFGR GFEEALHNLH TNSQAFPSAN

SAANSAANNT TAAAMTAVNN GISGGTFTYT NMTEGFSVIK DEPVNAASSP * jun 1

20

TVNPIDMEAQ EKIKLERKRQ RNRVAASKCR KRKLERISKL EDRVKVLKGE Basic Region

251

Figure 3. Genomic organization and molecular lesions of the jun locus. The genomic region containing the jun and the 14 3-3 $\xi$ genes (at band 46E on Drosophila chromosome II) is shown with landmark restriction sites. Jun and 14-3-3 transcripts are depicted with translated sequences (shaded). The area shown has been completely sequenced (EMBL database, accession no. Y12573). P-element insertion 07103 (obtained from the Berkeley Drosophila Genome Project as part of the Spradling collection) was mapped $2.8 \mathrm{~kb}$ to the $3^{\prime}$ end of the jun transcription unit. Imprecise excision of this $\mathrm{P}$ element yielded deficiency $E 73$, which removes the transcription start site and first exons of $14-3-3 \xi$ and the complete jun locus. jun ${ }^{1}$ represents an EMSinduced mutant allele that was isolated by its inability to complement the lethality of $E 73$. The jun ${ }^{1}$ open reading frame is prematurely terminated (asterisks), and can only be translated into a truncated gene product of 176 amino acids. The jun ${ }^{2}$ mutant was isolated originally, as 1(2)IA109, in a screen for embryonic lethal mutants (Nüsslein-Volhard et al. 1984). jun ${ }^{2}$ also originated from a nonsense mutation, this one in codon number 72 . The amino acid sequence of wild-type Jun with the location of the point mutations, the basic region, and the leucine zipper is shown at the bottom.

basic region and leucine zipper. Consequently, the gene product, if stably expressed at all, would no longer be able to dimerize and bind to DNA, causing loss of the jun function. Thus, jun ${ }^{1}$ can be regarded as a very strong and, possibly, a null allele. Immunostainings of jun mutant embryos with Jun antibodies revealed that jun ${ }^{1}$ is likely to be a null allele, as no anti-Jun immunoreactivity was detected (not shown).

Homozygous jun ${ }^{1}$ mutant embryos die in mid- to late embryogenesis with large anterior and dorsal holes, similar to the ones caused by bsk or hep (Fig. 4). Together with the experiments using dominant-negative forms of Jun, the jun ${ }^{1}$ phenotype confirms the role of Jun as a regulator of dorsal closure and, to a variable degree, of head involution. These defects are consistent with the

elevated Jun expression in the leading edge of the lateral epidermis during dorsal closure and along the cephalic furrow prior to and during head involution (Fig. 1). In characterizing $j u n^{1}$, we noticed similarities to a mutant that was originally isolated in a screen for embryonic lethal mutations in Drosophila (Nüsslein-Volhard and Wieschaus 1980; Nüsslein-Volhard et al. 1984). This mutant 1(2)IA109, like jun ${ }^{1}$, causes a dorsal closure defect and was genetically mapped to the same general region of chromosome IIR. Complementation analysis revealed that $j u n^{1}$ and l(2)IA109 are allelic, and sequence analysis showed that the 1(2)IA109 allele carries a stop codon at position 72 within the Jun open reading frame giving rise to a truncated translation product of only 71 amino acids (Fig. 3). Thus, 1(2)IA109 represents another jun allele, and we renamed it jun ${ }^{2}$. The phenotypes of $j u n^{1}$ and jun ${ }^{2}$ homozygous embryos, as well as those of embryos carrying various allelic combinations of $j u n^{1}, j u n^{2}$, and the E73 deficiency are all indistinguishable (Fig. 4). This confirms that the dorsal closure defect is a specific consequence of a lesion in jun and is not caused by another unknown mutation. Furthermore, it supports the assumption that jun ${ }^{1}$ and $j u n^{2}$ are null alleles.

To further characterize the dorsal closure defects caused by lack of Jun activity, mutant and wild-type embryos of stage 13 were stained with a marker to visualize cells at the leading edge of the lateral epidermis. Antibodies against the Coracle protein (Fehon et al. 1994)
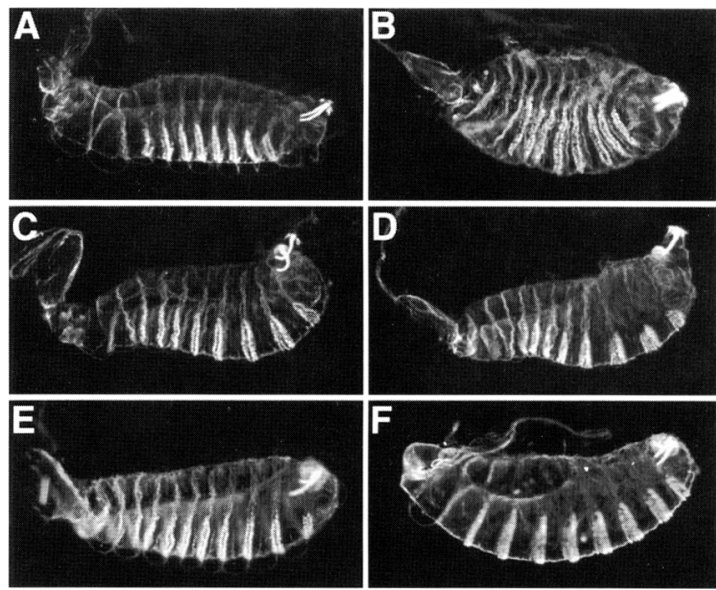

Figure 4. Embryonic phenotype of jun mutants. Cuticle preparations of the following genotypes are shown: $(A)$ iun $^{1} /$ iun $^{1}$; $(B)$ jun $^{2} /$ jun $^{2} ;(C)$ jun $^{1} / D f(2 R) E 73 ;(D)$ jun $^{2} / D f E 73$; (E) hep ${ }^{1} /$ hep $^{1}$; (F) $b s k^{1} / b s k^{1}$. All cuticles are from animals raised at $18^{\circ} \mathrm{C}$ except $b s k^{1}$ which was raised at $25^{\circ} \mathrm{C}$. For comparison a wild-type cuticle is shown in Fig. 2A. Note defects in dorsal closure that lead to a dorsally open cuticle. All allelic combinations of any jun alleles and the $E 73$ deficiency or homozygous deficiency $(A-D$; data not shown) have the same phenotype, which is very similar to the hep and bsk phenotypes $(E$ and $F$, respectively; $b s k^{1}$ is a temperature-sensitive hypomorph and displays slightly weaker defects). None of the mutants shows defects affecting the posterior structures, the Filzkörper. All embryos are oriented anterior left and dorsal up. 

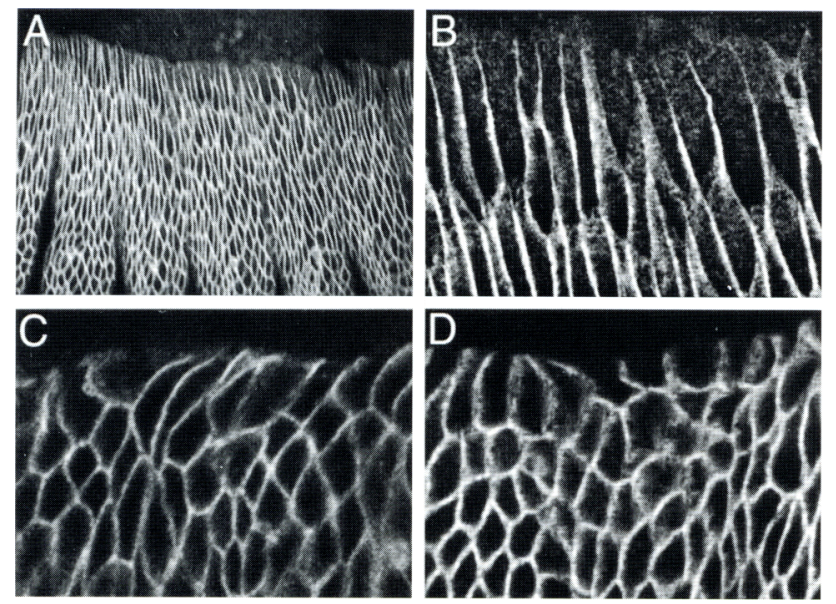

Figure 5. Cell shape changes during dorsal closure in wild-type and jun mutants as visualized with Coracle staining. All panels shown are derived from late stage 13 embryos (the embryos were staged according to their state of gut development). Anterior is left; dorsal up. $|A, B|$ Lateral epidermis of wild-type embryo ( $B$ is $4 \times$ magnification as compared to $A$ ): Note that cells have elongated along the dorsoventral axis, especially those of the leading edge (top cell layer). $(C, D)$ iun $^{1}$ and jun ${ }^{2}$ mutant embryos, respectively (shown at the same magnification as $B$ ): Note that the epidermal cells have not elongated.

outline individual epidermal cells and thus allow the detection of cell shape changes that take place during dorsal closure (Fig. 5). In wild type, dorsal closure initiates with a dorsoventral elongation of the cells at the leading edge of the lateral epidermis. The ventrally adjacent rows of epidermal cells subsequently undergo the same changes (Fig. 5A,B). In jun mutants the cells at the leading edge do not elongate to the same extent as in wild-type, and no cell shape changes are detectable more ventrally (Fig. C,D; data not shown). These data indicate that Jun is required for the initiation of dorsal closure, similar to hep and bsk.

\section{Jun in eye development}

The data described above provide evidence that the role of Jun in dorsal closure is that of a nuclear component in the Drosophila JNK signal transduction pathway. In the developing eye where we have studied the function of Drosophila Jun previously, the situation appears to be different. Here, Jun activity is regulated by a separate pathway that signals through Ras, Raf, and the ERK-type MAPK Rl (Biggs et al. 1994; Zipursky and Rubin 1994; Wassarman et al. 1995). Genetic and biochemical evidence illustrates that the phosphorylation of Jun by activated $\mathrm{Rl}$ can initiate neuronal differentiation in competent cells of the eye imaginal disc (Treier et al. 1995; Peverali et al. 1996).

To compare the role of Jun in photoreceptor differentiation versus dorsal closure, that is, as a target of ERK versus JNK signaling, we investigated the effect of jun mutant alleles on eye development. Clones of homozy- gous mutant eye tissue were induced in jun heterozygous animals by somatic recombination using $\mathrm{X}$ rays or the FRT-FLP system (Xu and Rubin 1993). Surprisingly, $j u n^{1}$, as well as jun ${ }^{2}$ homozygous clones were obtained and grow to a size that is comparable to the corresponding wild-type twin spots, indicating that the loss of Jun does not interfere with imaginal cell proliferation. Moreover, mutant clones look externally indistinguishable from surrounding wild-type tissue and show, upon microscopic inspection of tangential sections, only minor defects (Fig. 6). Most ommatidia contain the wild-type complement of photoreceptors, and photoreceptor cells are missing only occasionally. This very mild phenotype contrasts with the effect of dominant-negative Jun mutants that cause a more pronounced, yet still weakly penetrant, loss of photoreceptor cells (Bohmann et al. 1994, Treier et al. 1995).

The removal of Jun function by mutation or by expression of dominant-negative mutants has essentially the same detrimental effect on dorsal closure. In the eye, however, dominant-negative mutants interfere with photoreceptor differentiation more strongly than the complete removal of Jun. One possible explanation for this discrepancy is that Jun function in the Drosophila eye is redundant and a complete removal of the protein under otherwise wild-type conditions is largely without phenotypic consequences. The slightly stronger effect of the dominant-negative mutants could be rationalized if occupancy of the Jun-binding sites in the relevant target genes by mutated proteins has a more disruptive effect on their expression and on photoreceptor differentiation than no binding at all. To test this, we examined whether a reduction in the level of wild-type Jun activity would enhance the effect of expressing dominant negative $\left(s E\right.$-jun $\left.{ }^{A l a}\right)$ and gain-of-function $\left(s E\right.$-jun $\left.{ }^{A s p}\right)$ versions of Jun (Treier et al. 1995). We established Drosophila strains that express the modified Jun proteins in a heterozygous jun ${ }^{-}$background. Phenotypic analysis shows that the loss of photoreceptor phenotype caused by $s E$ -

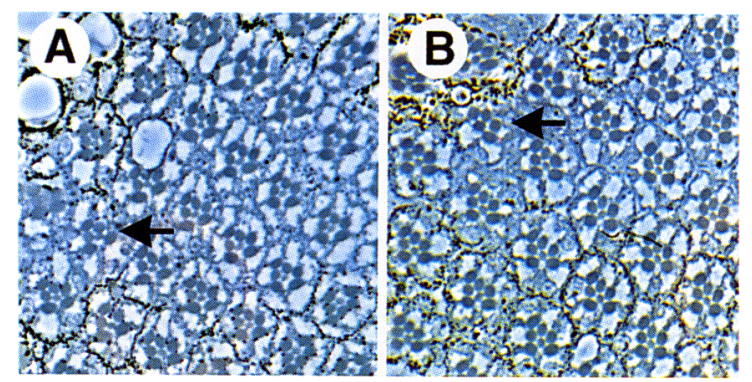

Figure 6. Clonal analysis of jun mutant eye phenotypes. Clones homozygous for the $\operatorname{jun}^{1}(A)$ and $j u n^{2}(B)$ alleles in adult eyes are marked by the absence of pigment. Anterior is left and dorsal up. Note that most mutant ommatidia have wild-type appearance with the typical trapezoidal arrangement of the six outer photoreceptor rhabdomeres and the central R7 (R8 is below plane of section). Occasionally one or two photoreceptors are lost in mutant ommatidia (marked by arrows in $A$ and $B$ ). The clone size is very similar to the wild-type twin clones (data not shown). 
$j u n^{A l a}$ is enhanced when it is combined with a $j u n^{1} /+$ or a iun $^{2} /+$ genotype (Fig. 7, A,B, and E,F). Similarly, the removal of one copy of jun increases the general disorder and number of ectopic photoreceptors in lines carrying $s E$-jun ${ }^{A s p}$ (Fig. 7C,D and G,H). These results indicate that the gain- and loss-of-function forms of Jun interfere specifically with the endogenously expressed wild-type protein. If the levels of the latter are decreased by halving the gene dose, the mutants can bind to the corresponding promoter elements more efficiently and thus their effects are stronger.

\section{Jun interacts genetically with the Sev/Ras/Raf/ERK signal transduction pathway}

The above results are consistent with there being several redundant downstream targets of the Ras/Raf/ERK signal transduction pathway in the developing eye. These should individually be able to instruct cells to adopt photoreceptor fate, so that under normal circumstances loss of one of them, in this case, Jun, can be compensated. To search for such a redundant function of Jun, we examined the effect of a reduced jun gene dose, that is, het-

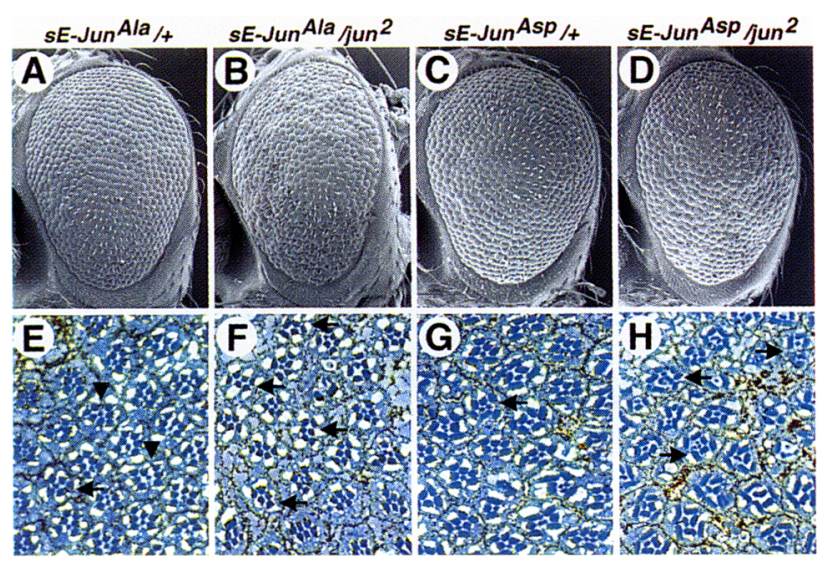

Figure 7. Enhancement of the dominant-negative, $s E-j u n^{A l a}$, and gain-of-function, $s E-j u n^{A s p}$, Jun phenotypes by gene dose reduction in jun. $(A-D)$ Scanning electron micrographs of adult eyes; and $(E-H)$ sections through such eyes. Anterior is left and dorsal up. $(A, E) s E-j u n^{A l a} /+;(B, F) s E-j u n^{A l a} / j^{2} n^{2} ;(C, G) s E-$ $j u n^{A s p} /+;(D, H) s E-j u n^{A s p} / j u n^{2}$. The dominant-negative $s E-j u-$ $n^{A l a}$ transgenic line used (no. 1) shows a weaker effect in wildtype than the line previously published (Treier et al. 1995): only a few ommatidia have lost one photoreceptor (an example is indicated by arrow) and some even contain an additional outer photoreceptor (arrowheads). Note that in the $s E-j u n^{A l a} / j u n^{2}$ genotype the number of ommatidia with missing photoreceptors is dramatically enhanced (examples indicated by arrows), and the presence of extra outer photoreceptors has disappeared as compared to $s E-j u n^{A l a} /+$. The gain-of-function $s E-j u n^{A s p}$ phenotype (presence of additional R7 photoreceptors, example shown by arrow) and the general disorder of the eye are both enhanced by gene dose reduction in $j u n\left(s E-j u n^{A s p} / j u n^{2}\right)$. Note that several ommatidia contain multiple extra R7 cells in $H$ (examples shown by arrows). Both jun ${ }^{1}$ and jun ${ }^{2}$ alleles have the same effect. erozygosity for the newly defined mutant jun alleles, in the context of either too much or too little signaling through the neurogenic Ras/Raf/ERK pathway.

In the first approach, the neurogenic signaling pathway in the developing eye was artificially activated by expression of gain-of-function alleles, including $\mathrm{sev}^{S 11}$, ras $^{\text {Val12 }}$, and $r^{\text {Ism }}$ (Sevenmaker), which lead to the characteristic appearance of ectopic photoreceptor cells (Basler et al. 1991; Dickson et al. 1992; Fortini et al. 1992; Brunner et al. 1994a). Under these conditions downstream targets of the pathway are expected to be ectopically hyperactivated and might become limiting in this way. This creates a "sensitized background" in which decreased concentrations of such targets can suppress the appearance of ectopic photoreceptor cells. This principle has been applied successfully in several genetic screens for downstream targets of signal transduction components (Chang et al. 1995; Dickson et al. 1995; Karim et al. 1996). When one copy of jun is removed from transgenic lines expressing the gain-of-function alleles mentioned above, a clear suppression of the mutant extra photoreceptor phenotype can be observed. Although the $\mathrm{ev}^{\mathrm{s11}}$ and $\mathrm{rl}^{\mathrm{sem}}$ alleles cause the appearance of supernumerary photoreceptors in nearly all ommatidia in a wild-type background, the removal of one copy of jun suppresses this effect. In animals carrying the activated alleles in a $j u n^{1} /+$ or $j u n^{2} /+$ background, approximately one quarter of the ommatidia revert to a wildtype appearance (Fig. 8A-F).

The Ellipse $e^{B 1}\left(E 1 p^{B 1}\right)$ gain-of-function allele of the Drosophila epidermal growth factor receptor (EGFR) represents another activated component of receptor tyrosine kinase (RTK)/Ras/ERK signaling (Baker and Rubin 1992). Both the eye and wing are affected in $E l p^{B 1}$, and ectopic wing vein tissue develops in the wing (Fig. 8G). Strikingly, the removal of one copy of jun suppresses this phenotypic defect almost completely (Fig. 8H). Thus, reduction in jun gene dose can be rate limiting in several independent RTK/Ras/ERK signaling events, implying a general role for Jun function downstream of this signaling cascade. Nevertheless, analysis of jun mutant clones in wing tissue does not reveal any phenotypic features normally detected with mutants of components of the EGFR/Ras/ERK-Rl pathway (data not shown). Thus, as in eye development, Jun function in the wing appears to be redundant.

Next, we examined the effect of reducing jun gene dosage in the context of a weakened differentiation signal. Decreased ERK activity as a result of heterozygosity of the $r l$ locus (genotype $r 1^{10 A} /+$ ) is phenotypically neutral with no detectable photoreceptor defects (Fig. 9A). Evidently, the system is flexible enough to accommodate moderate changes in signaling activity. However, if this genotype, $r 1^{10 A} /+$, is combined with one copy of a mutant jun allele or a jun deficiency $\left(\mathrm{jun}^{-} /+\right)$, we observe nonallelic noncomplementation and a strong phenotype (Fig. 9A-C). Now in most of the ommatidia one or more photoreceptors are missing. We conclude that upon reduction of $\mathrm{Rl}$ activity, downstream components are activated less strongly, and loss of even one jun copy can 
sevS11/t $\quad \operatorname{sev} S 11 /+; j u n^{1 /+}$
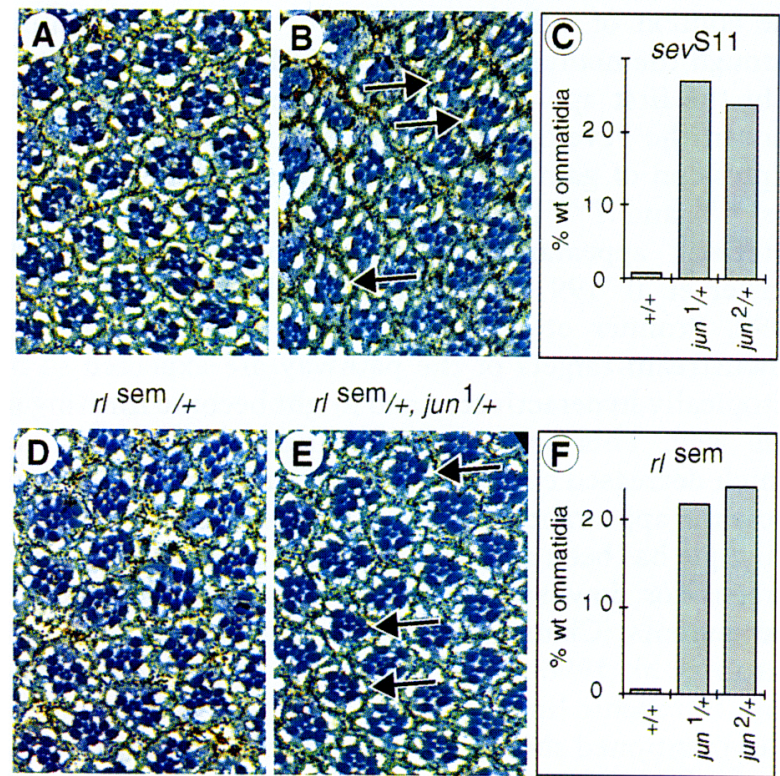

$$
E l p^{B 1} /+
$$

$E / p^{B 1 /+}, j u n^{2 /+}$

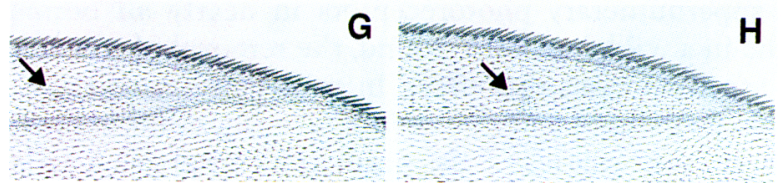

Figure 8. jun mutations dominantly suppress activated components of the RTK/Ras/Rl (ERK) pathway. $(A-F)$ Eye phenotypes of activated components of Sev/Ras/ERK signaling in the presence and absence of jun mutants. $(A, B, D, F)$ Sections through eyes of the respective genotypes; $(C, F)$ the statistical analysis. $(A-C)$ Gain-of-function Sev is suppressed by gene dose reduction in jun; $(A) \mathrm{sev}^{S 11} ;(B) \operatorname{sev}^{S 11}$; jun ${ }^{1} /+;(C)$ corresponding statistical analysis, percentage of wild-type ommatidia are shown (for each genotype at least three independent eyes and $>300$ ommatidia were analyzed). $(D-F)$ The gain-of-function allele of $r l / E R K$, Sem, is suppressed by gene dose reduction in jun; $(D) r l^{\mathrm{Sem}} ;(B) r l^{\mathrm{Sem}^{2}} ; \mathrm{jun}^{1} /+;(F)$ corresponding statistical analysis (as in $C$ ). Note that both jun alleles have the same effect. Examples of wild-type ommatidia are marked with arrows. $(G, H)$ The suppression effect of the wing phenotype of the gain-offunction allele of the Drosophila EGFR $(E l p)$ by gene dose reduction in jun. $(G) E l p^{B 1} /+$ (note the presence of extra wing vein tissue, marked by arrow); $(H)$ the wing has an almost wild-type appearance (note absence of extra vein tissue at arrow). In $88 \%$ of the $E 1 p^{B 1} /+$ wings significant extra L2 vein tissue was visible. This number drops to $22 \%$ when one copy of jun is mutated $\left(E^{B 1} p^{B 1} /\right.$ inn $\left.^{2}\right)$.

no longer be compensated by redundant factors anymore. The unusually strong phenotypic interaction of two heterozygous mutations unmasks the role of signal transduction from $\mathrm{Rl}$ to Jun in photoreceptor differentiation. The dominant interaction between $\mathrm{Rl}$ and Jun is not a peculiarity of this combination of alleles. Similar conclusions can be drawn from the interaction of mutant jun alleles with weak mutations elsewhere in the path- way. For example, in the $\operatorname{sev}^{E 4}$; $\operatorname{Sos}^{J C 2} /+$ genotype, Sevmediated signaling is reduced because of a mutation in sev that is only partially rescued by a gain-of-function mutation in the GDP/GTP exchange factor Son of sevenless (Sos) (Rogge et al. 1991). Under these conditions, activation of Ras is impaired, causing loss of R7 photoreceptors in a fraction of the ommatidia. This weak defect is significantly enhanced when the jun locus is heterozygous mutant (Fig. 9D-F).

The enhancement of the $\operatorname{sev}^{E 4} ; \operatorname{Sos}^{I C 2} /+$ phenotype and, particularly, the striking synthetic mutant phenotype of $j u n / r 1^{10 a}$ unmasks the otherwise redundant role of Jun in the determination of photoreceptor cell fate and documents the relevance of the Rl-Jun interaction in the process.

\section{Discussion}

The identification of the mutant Drosophila jun alleles described in this report has made it possible to dissect the signaling and regulatory functions of Jun in a developing organism. Consistent with a wealth of data obtained in tissue culture experiments, Jun acts pleiotropically and influences morphogenesis and cell differentia-

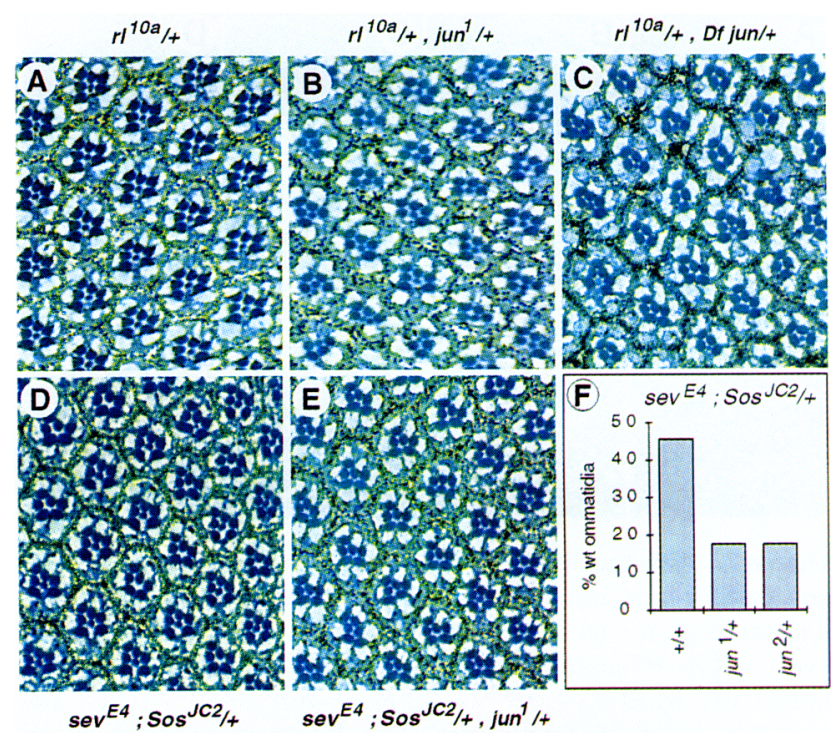

Figure 9. Mutations in iun are limiting components of photoreceptor induction when Sev/Ras/Rolled(ERK) signaling is reduced. All panels show eye sections of the respective genotypes, except $F$, which shows a statistical analysis. $(A-C)$ A combination of $r l /+$ and $j u n /+$ mutations reveals nonallelic noncomplementation between both genes $\left[(B) r 1^{10 a} /+, j u n^{2} /+;(C) r 1^{10 a} /+\right.$, $D f($ jun $) /+]$, whereas both single heterozygotes, $r I^{10 a} /+|A|$ and the jun alleles over wild type (not shown) are completely wild type. The flies of the genotypes shown in $B$ and $C$ have also some missing bristles (not shown). (D-F) The phenotype of a combination of hypomorphic sev and Sos alleles is enhanced by gene dose reduction in jun. $(D) \mathrm{sev}^{E 4}$; $\operatorname{Sos}^{J C 2} /+$. $(E) \mathrm{sev}^{E 4}$; $\operatorname{SoS}^{I C 2} /+$, jun $^{1} /+$. $(F)$ statistical analysis of the respective genotypes (percentage of mutant wild-type ommatidia are shown; analysis as in Fig. 7). 
tion in various developmental situations. Previous results that had implicated Jun in the determination and differentiation of photoreceptor cells in the developing eye were confirmed and extended. Moreover, we could demonstrate the role of Jun in the regulation of dorsal closure in the embryo and of wing morphogenesis during pupal stages.

In addition to providing new insight into the molecular basis of Drosophila development, these studies revealed interesting properties of Jun signaling in an intact organism: Jun can have both redundant and nonredundant functions during development, and it can act as a recipient of distinct signal transduction pathways.

\section{Redundant and nonredundant functions of Jun}

Jun is essential in embryonic development. Loss of Jun function in transgenic lines expressing dominant-negative mutants, or in animals that are homozygous for mutant jun alleles, causes lethality with defects in dorsal closure and in head involution. The demonstration that jun is essential for dorsal closure, even though not unexpected, based on suggestive biochemical evidence (Riesgo-Escovar et al. 1996; Sluss et al. 1996), is not a trivial result. In spite of its name, JNK has been shown in mammalian systems to phosphorylate a number of transcription factors besides Jun, and the INK-Jun signaling connection has never before been implicated in the control of developmental processes or cell shape changes.

In contrast to the situation in the embryo, removal of Jun is much less disruptive in eye development, at least under otherwise wild-type conditions. In jun-deficient eye tissue, ommatidial assembly and photoreceptor differentiation are almost normal and only infrequent mild defects can be observed. However, when the Ras/ERK signaling pathway, which instructs the pluripotent imaginal disc cells to assume a neuronal fate, is disturbed, Jun function becomes critical for this process. Strikingly, a reduction of jun gene dose by $50 \%$ causes defects in most ommatidia in heterozygous ERK null mutants. Such nonallelic noncomplementation is observed only rarely in Drosophila and usually only between two genes acting in the same pathway, for example, some $d s h /+; w g /+$ double heterozygotes display defects in the distal wing blade (Theisen et al. 1994). These results, together with the finding that mutant jun alleles give rise to significant loss of photoreceptor differentiation in sensitized genetic backgrounds, as well as biochemical evidence (Peverali et al. 1996), clearly document a function of Jun in the Ras/ERK signaling pathway that specifies photoreceptor cell fate (Fig. 10). This function appears however to be redundant in wild type flies.

Redundant functions of Jun are not unprecedented. In the mouse, null mutations of c-iun, the mammalian homolog of Drosophila jun, have an unexpectedly mild phenotype and cause death only late in embryogenesis (Hilberg et al. 1993; Johnson et al. 1993). It was concluded that some of the functions of Jun in the mouse are redundant, and lack of c-Jun can be compensated by

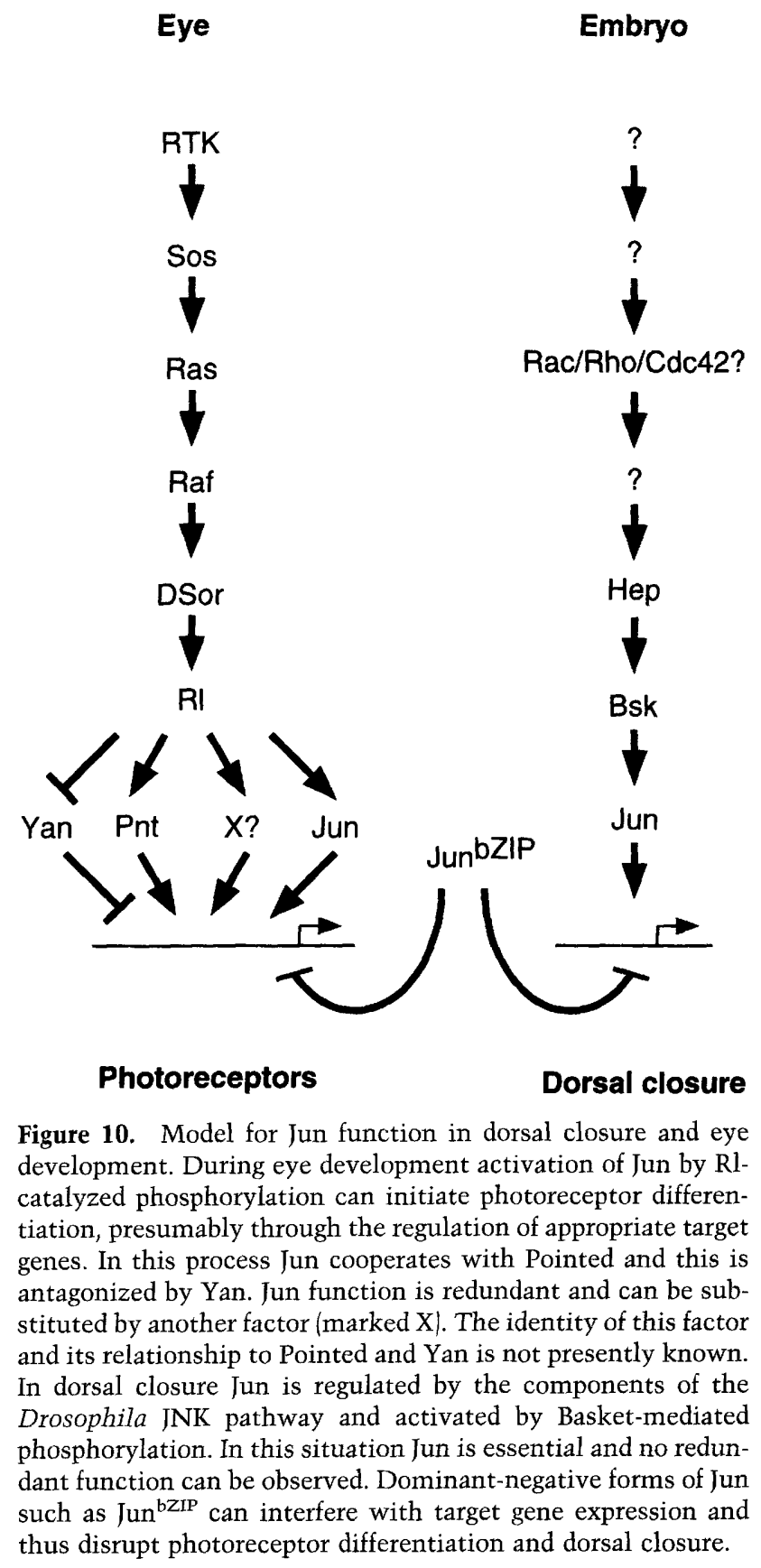

other gene products. Genetic redundancy is a phenomenon that is widespread in biology and has been found in eukaryotic organisms from yeast to mammals. The significance, the biological function, and the reason for the evolutionary stability of apparently redundant groups of genes are much debated topics. Our findings suggest that the redundant role of jun in photoreceptor differentiation serves to make eye development a precise and reliable process that is not easily disrupted. In contrast to many sensitized mutant phenotypes, the development of a wild-type eye is fairly insensitive to genetic or environmental disturbances, such as heterozygosity of important regulatory genes or temperature fluctuations. We 
suggest that this robust fidelity of wild-type development is ensured by the action of several factors that have the same or very similar regulatory function and thus back each other up. Our results support this model as they show that Jun function becomes important when disturbances in the normal determination and differentiation program (such as a reduction in ERK activity because of a loss of one allele) need to be compensated (Fig. $10)$.

The question of which factors act redundantly with Jun cannot be answered based on the available results. It can be assumed that other ERK-responsive transcription factors could fulfill this function. Obvious candidates include Pointed P2 and Yan, which have been shown to be regulated by $\mathrm{Rl}$ phosphorylation and can cooperate with Jun in photoreceptor determination (Brunner et al. 1994b; O'Neill et al. 1994; Rebay and Rubin 1995; Treier et al. 1995) (Fig. 10). A more tentative possibility is Drosophila Fos, which can bind to DNA independently of Jun (Perkins et al. 1988) and is expressed in the developing eye (J. Zeitlinger, unpubl.).

Drosophila Jun is a target for both JNK and ERK signal transduction

There has been a certain controversy of whether Jun is regulated by ERK or by INK (Minden et al. 1994). The results presented here, together with previously published biochemical data, indicate that Jun can be regulated by both types of MAPK signal transduction pathways. During eye development, and probably in the wing, Jun is phosphorylated by the ERK-type MAPK Rl, whereas in dorsal closure Jun is a target for the JNK-type enzyme Bsk (Fig. 10).

Thus, Jun acts as a convergence point for several signals and signal transduction pathways. Similar multiple responsiveness has also been reported in the case of other inducible transcription factors such as CREB or Elk. In tissue culture these factors are targets of more than one signal transduction pathway, and multiple kinases can phosphorylate them to modulate their activity (Dash et al. 1991; Lalli and Sassone-Corsi 1994; Gille et al. 1995; Whitmarsh et al. 1995; Treier and Bohmann 1996). A number of interesting questions arise from the concept of several signal transduction pathways converging on a single transcription factor: How is the specificity of different signal responses determined if they converge on the same "bottleneck", for example, Jun phosphorylation? Do Bsk and Rl have equivalent biochemical and regulatory effects? Can the two pathways act in the same cells, and if so, do they cooperate? In this regard it is worth pointing out that in light of current data it is not clear whether Bsk has a function in eye development. $b s k^{-}$clones, like jun ${ }^{-}$clones, show essentially no mutant phenotype (Riesgo-Escovar et al. 1996), but it is conceivable that Bsk, which is expressed in the eye (Sluss et al. 1996), has a redundant function similar to that of Jun. These and related questions can now be addressed.

To better understand the role of Jun in Drosophila development, more has to be learned about the upstream regulators and downstream targets of the transcription factor. Experiments with dominant-negative mutants point to Cdc42 and Rac-related proteins as potential upstream activators of the Hep/Bsk cascade in dorsal closure (Harden et al. 1995; Riesgo-Escovar et al. 1996). It will be important to determine how these proteins fit into the pathway, whether Jun activation by Rac or Cdc42 has a more general function in Drosophila development, and how these small G-proteins are themselves activated.

At this time we can only speculate about downstream targets of Jun. In dorsal closure the gene puckered is expressed with a pattern and a responsiveness that is consistent with it being a target gene of Jun (Ring and Martinez-Arias 1993; Glise et al. 1995). Another group of mutations affecting dorsal closure belong to the Decapentaplegic (Dpp) pathway. Certain alleles of $d p p$, as well as punt and thick veins $(t k v)$ mutants cause a dorsal open phenotype (Letsou et al. 1995; Ruberte et al. 1995). It will be interesting to investigate whether there is a connection between these genes and the Bsk/Jun signaling pathway.

\section{Materials and methods}

\section{Immunohistochemistry}

Antibody staining was carried out according to standard methods using $0.1 \%$ Tween. The affinity-purified antibody against Jun (Bohmann et al. 1994; Peverali et al. 1996) was diluted 1: 4000 and preincubated (Fig. 1) and 1:2000 for jun and bsk mutant embryos. The anti-Coracle antibody (Fehon et al. 1994) was used 1:5000. To identify homozygous mutant embryos, jun alleles were balanced over a $w g-l a c Z C y O$ marked balancer, and embryos were costained with a commercial anti- $\beta$-galactosidase antibody (Cappel). Coracle staining was detected using the European Molecular Biology Laboratory (EMBL) confocal microscope.

\section{Genetics}

The deficiency $D f(2 R) E 73$ was obtained by mobilizing $P\left(\mathrm{ry}^{+}\right] 1(2) 07103 / \mathrm{CyO}$ by crossing with $S p / C y O ; S b, \Delta 2-3 /$ $T M 6, U b x$ and subsequent $F_{2}$ screening for $x y$ offspring which should include imprecise excisions of the P element. Established stocks were analyzed for genomic lesions by Southern blot and polytene chromosome in situ hybridization using genomic probes of the jun locus.

About 1000 isogenized $a l, d p, b w, s p$-marked males were mutagenized by feeding with $1 \%$ succrose $25 \mathrm{~mm}$ EMS solution for $8 \mathrm{hr}$. Chromosomes were extracted by mating to a $\mathrm{CyO}$ balancer stock. Male $F_{1}$ progeny (13183) were individually crossed to $D f(2 R) E 73 / C y O$ females. $F_{2}$ offspring were scored for noncomplementation, and stocks of these fly lines were established. All obtained lethals fall into three complementation

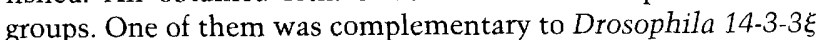
mutants. The jun ${ }^{1}$ allele was cloned by PCR and sequenced by the EMBL sequencing facility.

\section{Drosophila strains}

The activated jun allele $s E-j u n^{A s p}$ is described in Treier et al. (1995). Dominant-negative $s E-j u n^{A l a}$ stocks were reestabished. The UAS-jun ${ }^{b Z I P}$ fly strain was obtained by cloning the basic 
region and leucine zipper (amino acids 183-289) of Drosophila Jun into the P-element transformation vector pUAST (Brand and Perrimon 1993), followed by germ-line transformation using standard methods (Spradling and Rubin 1982). The Gal4 drivers used in this study are described in Brand and Perrimon (1993). The following fly strains of sensitized or activated alleles of the receptor tyrosine kinase signaling pathway have been used: $\operatorname{sev}^{\text {S11 }}$ (Basler et al. 1991); $r l^{\text {Sem }}$ (Brunner et al. 1994a); $s E-$ ras $^{V 12}$ (Fortini et al. 1992); Elp ${ }^{B 1}$ (Baker and Rubin 1992); $\mathrm{rl}^{10 a}$ and $\mathrm{SeV}^{E 4} ; \operatorname{sos}^{I C 2}$ (Rogge et al. 1991; Biggs et al. 1994). The isogenized $a l, d p, b w, s p$ was a gift from Cristiana Molinari(EMBL) and Slava Bolshakov (EMBL).

\section{Acknowledgments}

We thank David Jackson for the UAS-Jun ${ }^{\text {bZIP }}$ P-element lines and Fiorenzo Peverali for Jun antibodies. We are most grateful to Anna Cyrklaff and Ursula Weber for help with chromosome in situ hybridization, and Roger Wepf for skilled assistance with the scanning electron microscope analysis. We thank Steve Hou and Norbert Perrimon for sharing unpublished results, Slava Bolshakov, Cristiana Molinari, Rick Fehon, Ernst Hafen, Stephane Noselli, Gerald Rubin, Gerd Vorbrüggen, Herbert Jäckle, Larry Zipursky, and the Tübingen Stock Center for fly strains and reagents, and members of the Bohmann and Mlodzik labs for discussion and advice. Nick Brown, Suzanne Eaton, and Iain Mattaj made helpful comments on the manuscript.

The publication costs of this article were defrayed in part by payment of page charges. This article must therefore be hereby marked "advertisement" in accordance with 18 USC section 1734 solely to indicate this fact.

\section{References}

Angel, P. and M. Karin. 1991. The role of Jun, Fos and the AP-1 complex in cell proliferation and transformation. Biochim. Biophys. Acta 1072: 129-157.

Armstrong, R.C. and M.R. Montminy. 1993. Transsynaptic control of gene expression. Annu. Rev. Neurosci. 16: 17-29.

Baker, N.E. and G.M. Rubin. 1992. Ellipse mutations in the Drosophila homologue of the EGF receptor affect pattern formation, cell division and cell death in eye imaginal discs. Dev. Biol. 150: 381-396.

Basler, K., B. Christen, and E. Hafen. 1991. Ligand-independent activation of the sevenless receptor tyrosine kinase changes the fate of cells in the developing Drosophila eye. Cell 64: 1069-1081.

Biggs, W.H.I., K.H. Zavitz, B. Dickson, A. van der Straten, D. Brunner, E. Hafen, and S.L. Zipursky. 1994. The Drosophila rolled locus encodes a MAP kinase required in the sevenless signal transduction pathway. EMBO J. 13: 1628-1635.

Bohmann, D., M.C. Ellis, L.M. Staszewski, and M. Mlodzik. 1994. Drosophila Jun mediates Ras-dependent photoreceptor determination. Cell 78: 973-986.

Brand, A.H. and N. Perrimon. 1993. Targeted gene expression as a means of altering cell fates and generating dominant phenotypes. Development 118: 401-415.

Brunner, D., N. Oellers, J. Szabad, W.H.I. Biggs, L.H. Zipursky, and E. Hafen. 1994a. A gain-of-function mutation in drosophila MAP kinase activates multiple receptor tyrosine kinase signaling pathways. Cell 76: 875-888.

Brunner, D., K. Dücker, N. Oellers, E. Hafen, H. Scholz, and C. Klämbt. 1994b. The Ets-domain protein pointed P2 is a target of MAP kinase in the sevenless signal transduction pathway. Nature 370: 386-389.
Campos-Ortega, J.A. and V. Hartenstein. 1985. The embryonic development of Drosophila melanogaster. Springer Verlag, Berlin, Germany.

Chang, H.C., N.M. Solomon, D.A. Wassarman, F.D. Karim, M. Therrien, G.M. Rubin, and T. Wolff. 1995. phyllopod functions in the fate determination of a subset of photoreceptors in Drosophila. Cell 80: 463-472.

Darnell, J.E., Jr., I.M. Kerr, and G.R. Stark. 1994. Jak-STAT pathways and transcriptional activation in response to IFNs and other erxtracellular signalling proteins. Science 264: 14151421.

Dash, P.K., K.A. Karl, M.A. Colicos, R. Prywes, and E.R. Kandel. 1991. cAMP response element-binding protein is activated by $\mathrm{Ca}^{2+} /$ calmodulin- as well as cAMP-dependent protein kinase. Proc. Natl. Acad. Sci. 88: 5061-5065.

Davis, R.J. 1994. MAPKs: New JNK expands the group. Trends Biochem. Sci. 19: 470-473.

Dérijard, B., M. Hibi, I. Wu, T. Barrett, B. Su, T. Deng, M. Karin, and R. Davis. 1994. JNK1: A protein kinase stimulated by UV-light and $\mathrm{Ha}-\mathrm{Ras}$ that binds and phosphorylates the c-Jun activation domain. Cell 76: 1025-1037.

Dickson, B., F. Sprenger, D. Morrison, and E. Hafen. 1992. Raf functions downstream of Ras1 in the sevenless signal transduction pathway. Nature 360: 600-603.

Dickson, B.J., M. Dominguez, A. van der Straaten, and E. Hafen. 1995. Control of Drosophila photoreceptor cell fates by Phyllopod, a novel nuclear protein acting downstream of the Raf kinase. Cell 80: 453-462.

Fehon, R.G., I.A. Dawson, and T.S. Atravanis. 1994. A Drosophila homologue of membrane-skeleton protein 4.1 is associated with septate junctions and is encoded by the coracle gene. Development 120: 545-557.

Fortini, M.E., M.A. Simon, and G.M. Rubin. 1992. Signalling by the sevenless protein tyrosine kinase is mimicked by Ras1 activation. Nature 355: 559-561.

Gille, H., T. Strahl, and P.E. Shaw. 1995. Activation of ternary complex factor Elk-1 by stress-activated protein kinases. Curr Biol 5: 1191-2000.

Glise, B., H. Bourbon, and S. Noselli. 1995. hemipterous encodes a novel Drosophila MAP kinase kinase, required for epithelial cell sheet movement. Cell 83: 451-461.

Greenwald, I. and G.M. Rubin. 1992. Making a difference: The role of cell-cell interactions in establishing seperate identities for equivalent cells. Cell 68: 271-282.

Harden, N., H.Y. Loh, W. Chia, and L. Lim. 1995. A dominant inhibitory version of the small GTP-binding protein Rac disrupts cytoskeletal structures and inhibits developmental cell shape changes in Drosophila. Development 121: 903914.

Herskowitz, I. 1995. MAP kinase pathways in yeast: For mating and more. Cell 80: 187-197.

Hilberg, F., A. Aguzzi, N. Howells, and E.F. Wagner. 1993. c-jun is essential for normal mouse development and hepatogenesis. Nature 365: 179-181.

Johnson, R.S., B. van Lingen, V.E. Papaioannou, and B.M. Spiegelman. 1993. A null mutation at the c-jun locus causes embryonic lethality and retarded cell growth in culture. Genes \& Dev. 7: 1309-1317.

Karim, F.D., H.C. Chang, M. Therrien, D.A. Wassarman, T. Laverty, and G.M. Rubin. 1996. A screen for genes that function downstream of Ras1 during Drosophila eye development. Genetics 143: 315-329.

Knust, E. 1996. Drosophila morphogenesis: Follow-my-leader in epithelia. Curr. Biol. 6: 379-381.

Kockel, L., G. Vorbrüggen, H. Jäckle, M. Mlodzik, and D. Boh-

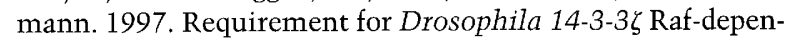




\section{Kockel et al.}

dent photoreceptor development. Genes \& Dev. 11: 11401147.

Kyriakis, J.M., P. Banerjee, E. Nikolakaki, T. Dai, E.A. Rubie, M.F. Ahmad, J. Avruch, and J.R. Woodgett. 1994. The stressactivated protein kinase subfamily of c-Jun kinases. Nature 369: 156-160.

Lalli, E. and P. Sassone-Corsi. 1994. Signal transduction and gene regulation: The nuclear response to cAMP. I. Biol. Chem. 269: 17359-17362.

Letsou, A., K. Arora, J.L. Wrana, K. Simin, V. Twombly, J. Jamal, K. Staeheling-Hampton, F.M. Hofmann, W.M. Gelbart, J. Massagué, and M.B. O'Connor. 1995. Drosophila Dpp signaling is mediated by the punt gene product: A dual ligandbinding type II receptor of the TGF- $\beta$ receptor family. Cell 80: 899-908.

Marshall, C.J. 1994. MAP kinase kinase kinase, MAP kinase kinase and MAP kinase. Curr. Opin. Genet. Dev. 4: 82-89.

- 1995. Specificity of tyrosine kinase signaling: Transient versus sustained extracellular kinase activation. Cell 80: 179-185.

Minden, A., A. Lin, T. Smeal, B. Derijard, M. Cobb, R. Davis, and M. Karin. 1994. c-Jun N-terminal phosphorylation correlates with activation of the JNK subgroup but not the ERK subgroup of mitogen-activated protein kinases. Mol. Cell. Biol. 14: 6683-6688.

Nüsslein-Volhard, C. and E. Wieschaus. 1980. Mutations affecting segment number and polarity in Drosophila. Nature 287: 795-801.

Nüsslein-Volhard, C., E. Wieschaus, and M. Kluding. 1984. Mutations affecting the pattern of the larval cuticle in Drosophila melanogaster I. Zygotic loci on the second chromosome. Wilhelm Roux's Arch. Dev. Biol. 193: 235-271.

O'Neill, E.M., I. Rebay, R. Tjian, and G.M. Rubin. 1994. The activities of Ets-related transcription factors required for Drosophila eye development are modulated by the Ras/ MAPK pathway. Cell 78: 137-147.

Perkins, K.K., G.M. Dailey, and R. Tjian. 1988. Novel Jun- and Fos-related proteins in Drosophila are functionally homologous to enhancer factor AP-1. EMBO 1. 7: 4265-4273.

Peverali, F.A., A. Isaksson, A.G. Papavassiliou, L.M. Staszewski, M. Mlodzik, and D. Bohmann. 1996. Phosphorylation of Drosophila Jun by the MAP kinase Rolled during R7 photoreceptor differentiation. EMBO. I. 15: 3943-3950.

Rebay, I. and G.M. Rubin. 1995. Yan functions as a general inhibitor of differentiation and is negatively regulated by activation of the Ras1/MAPK pathway. Cell 81: 857-866.

Riesgo-Escovar, J.R., M. Jenni, A. Fritz, and E. Hafen. 1996. The Drosophila Jun-N-terminal kinase is required for cell morphogenesis but not for DJun-dependent cell fate specification in the eye. Genes \& Dev. 10: 2759-2768.

Ring, J.M. and A. Martinez-Arias. 1993. puckered, a gene involved in position-specific cell differentiation in the dorsal epidermis. Development 119: 251-259.

Rogge, R.D., C.A. Karlovich, and U. Banerjee. 1991. Genetic dissection of a neurodevelopmental pathway: son of sevenless functions downstream of the sevenless and EGF receptor tyrosine kinases. Cell 64: 39-48.

Ruberte, E., T. Marty, D. Nellen, M. Affolter, and K. Basler. 1995. An absolute requirement for both the type II and type I receptors, punt and thick veins, for Dpp signaling in vivo. Cell 80: 889-897.

Siebenlist, U., G. Franzoso, and K. Brown. 1994. Structure, regulation and function of NFkB. Annu. Rev. Cell. Biol. 10: 405455.

Sluss, H.K., Z. Han, T. Barrett, R.J. Davis, and Y.T. Ip. 1996. A JNK signal transduction pathway that mediates morphogen- esis and an immune response in Drosophila. Genes \& Dev. 10: $2745-2758$.

Spradling, A.C. and G.M. Rubin. 1982. Transposition of cloned P-elements into Drosophila germline chromosomes. Science 218: 341-347.

Theisen, H., J. Purcell, M. Bennett, D. Kansagara, A. Syed, and J.L. Marsh. 1994. dishevelled is required during wingless signalling to establish both cell polarity and cell identity. Development 120: 347-360.

Treier, M. and D. Bohmann. 1996. Transcription factor phosphorylation. In Protein phosphorylation (ed. F. Marks), pp. 297-327. Verlag Chemie, Weinheim, Germany.

Treier, M., D. Bohmann, and M. Mlodzik. 1995. Jun cooperates with the Ets-domain protein Pointed to induce photoreceptor R7 fate in the Drosophila eye. Cell 83: 753-760.

Wassarman, D.A., M. Therrien, and G.M. Rubin. 1995. The Ras signaling pathway in Drosophila. Curr. Opin. Genet. Dev. 5: $44-50$.

Whitmarsh, A.J., P. Shore, A.D. Sharrocks, and R.J. Davis. 1995. Integration of MAP kinase signal transduction pathways at the serum response element. Science 269: 403-407.

$\mathrm{Xu}, \mathrm{T}$. and G.M. Rubin. 1993. Analysis of genetic mosaics in developing and adult Drosophila tissues. Development 117: 1223-1237.

Zipursky, S.L. and G.M. Rubin. 1994. Determination of neuronal cell fate: Lessons from the R7 neuron of Drosophila. Annu. Rev. Neurosci. 17: 373-397. 


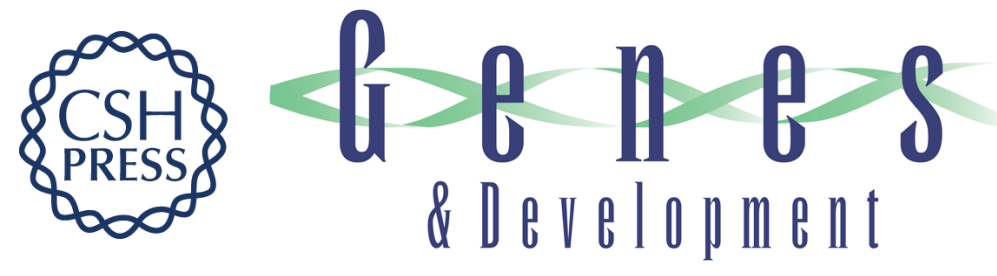

\section{Jun in Drosophila development: redundant and nonredundant functions and regulation by two MAPK signal transduction pathways.}

L Kockel, J Zeitlinger, L M Staszewski, et al.

Genes Dev. 1997, 11:

Access the most recent version at doi:10.1101/gad.11.13.1748

References This article cites 53 articles, 17 of which can be accessed free at:

http://genesdev.cshlp.org/content/11/13/1748.full.html\#ref-list-1

License

Email Alerting

Service

Receive free email alerts when new articles cite this article - sign up in the box at the top right corner of the article or click here.

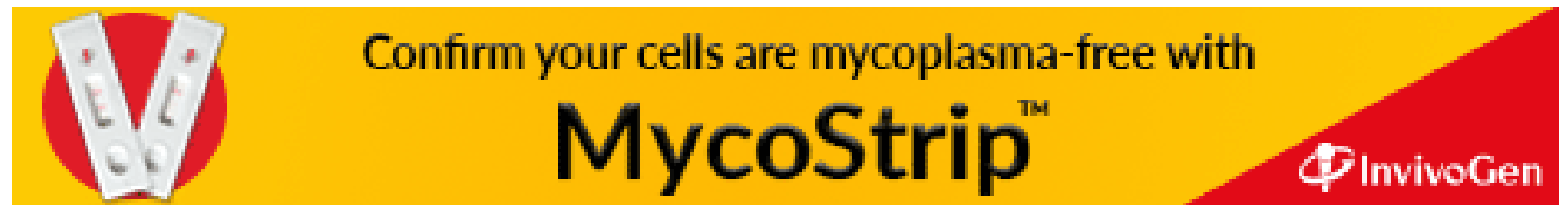

